\title{
Dynamics of Inverter-Based Resources in Weak Distribution Grids
}

\author{
Mehdi Ghazavi Dozein, Student Member, Bikash C. Pal, Fellow, IEEE, Pierluigi Mancarella, Senior \\ Member, IEEE
}

\begin{abstract}
This work presents the modelling fundamentals to study the dynamics of inverter-based resources (IBRs) in weak distribution grids and derive disturbance performance characteristics describing how they should behave under different conditions. More specifically, with respect to smallsignal disturbances we study the possible voltage violations following frequency response from IBRs due to low system strength and also propose specific design requirements for IBR $d$ $q$ current control to guarantee a stable response. In the context of large-signal disturbances, we highlight how active power-voltage control may not be effective due to a delay imposed by the physical features of the distribution network. Further, we mathematically discuss how IBR reactive power control could enhance its phase-locked loop stability. The proposed disturbance performance characteristics are then integrated into the IBR converter control via a novel voltage-priority reference generation strategy. Simulation results on a real Australian network show the efficacy of the proposed operational and control design requirements, and highlight possible unexpected active/reactive power interactions in weak distribution grids.
\end{abstract}

Index Terms - Disturbance performance characteristics, Dynamic supports, IBR stability, Inverter-based resources, System strength, Weak distribution grids.

\section{INTRODUCTION}

A ctive distribution networks (ADNs) with many inverterbased resources (IBRs) are potential candidates for the provision of flexibility and system services. These will be more needed in low-carbon grids that may be prone to frequency instability under low-inertia conditions [1-2] and to voltage instability at weak grid connections due to reduction in short-circuit level [3]. Most previous works have focused on the capabilities of inverter-based technologies (e.g., photovoltaic (PV) units [4], battery energy storage systems [56]) in providing voltage/reactive and frequency/active dynamic supports in transmission systems [4-6] or DC/AC micro-grids [7]. In the context of distribution grids, previous investigations can mainly be categorized into five groups aimed to: 1) alleviate overvoltage problems in PV-rich distribution feeders through active/reactive power control of converters [8-9] or coordination of PV units with distributed

This work was supported in part by AusNet Services.

M. Ghazavi Dozein and P. Mancarella are with the School of Electrical and Electronic Engineering, The University of Melbourne, Australia. (E: \{mehdi.ghazavidozein, pierluigi.mancarella\}@unimelb.edu.au). P. Mancarella is also with the University of Manchester, Manchester, UK (E: p.mancarella@manchester.ac.uk). B. C. Pal is with the Department of Electrical and Electronic Engineering, Imperial College London, UK (E. b.pal@imperial.ac.uk) batteries [10-11]; 2) boost the low-voltage ride-through capability of inverter-based units, in particular distributed PV units [12], through different mechanisms ranging from oversizing the converter to fine-tuning an inductive filter at the IBR terminals [12-14]; 3) highlight possible interactions of distribution network control with transmission grid control to improve voltage stability [15]; 4) focus on dynamic equivalencing of ADNs to reduce the difficulty in combined distribution-transmission dynamic modelling for frequency response analysis [16]; 5) quantify the aggregate capabilities of distributed resources in various system support services through $P-Q$ flexibility maps based on steady-state analysis [17-18]. From our analysis of the literature, there is no modelling foundations to study IBR dynamics in weak distribution grids, IBR potential benefits in providing multiple dynamic supports, and the associated technical requirements and challenges. In this context, a weak distribution grid refers to a distribution network characterized by: a connection point to the upstream transmission grid with relatively low shortcircuit level (e.g., a short-circuit ratio smaller than three); nonnegligible resistive components; high sensitivities in voltages and angles with respect to the change in active/reactive power; and a strong coupling in angle-voltage-current dynamics. Generally speaking, two points are important when it comes to stability analysis of grid-connected inverter-based resources: 1) The existence of the post-contingency equilibrium point: most previous works have aimed to study whether an equilibrium point exists, for system voltages and IBR state variables, following grid disturbances (e.g., [19]). 2) The IBR dynamic behavior: this indicates how an IBR emerges to its post-contingency equilibrium point. In fact, it is also of interest to study how IBR dynamic behavior (active/reactive power control) can potentially affect the external system voltage/frequency/angle dynamics, how external system physical features may impact the stable IBR dynamic behavior, and how IBR active/reactive power control may affect its own converter control operation and synchronism. This point is even more important when it comes to weak grid connections due to high sensitivities in system variables, the potential problems with IBR synchronism, etc. In particular, we have identified the following research gaps that we aim to bridge:

i) While previous works have mainly addressed steady-state or quasi steady-state aspects (e.g., volt-var control) of distribution grid operation, IBR dynamic aspects and challenges associated with the strength of a distribution grid and its physical features have not been investigated at all. This 
is key to understand how to define disturbance performance characteristics of converter-based resources in weak distribution networks. In this regard, disturbance performance characteristics refer to the diagrams indicating what dynamic behavior IBRs should have when there is no system disturbance as well as in the presence of small-signal disturbances or large-signal disturbances. This is a research gap since all existing guidelines ignore the physical features of weak distribution feeders.

ii) There is no clear understanding on how dynamic supports from IBRs in weak distribution grids may be limited due to system physical features (e.g., low system strength) and gridcode requirements (e.g., angle jump requirement) in addition to the widely known converter-level control instability, e.g., phase-locked loop (PLL) instability [3]. Moreover, there is no work to differentiate whether IBR inability to provide system dynamic support might emerge due to their converter-level instabilities or external system physical features.

iii) IBR dynamic response to simultaneous voltage/frequency disturbances, which are prone to happen in weak grids [3], has not been studied at all. In particular, the impacts of IBR active/reactive power control/priority on their converter stability as well as on the system stability have not been well explored.

iv) It is not clear how frequency control from IBRs may result in voltage violations and how this phenomenon relates to the low system strength at the distribution-transmission point of interface (DTPOI). In addition, it is necessary to understand how physical characteristics of weak distribution grids may necessitate specific design requirements on IBR converter control, e.g., PLL and $d-q$ current controllers.

On the above premises, this work, for the first time, presents fundamental mathematical discussions on the dynamic behavior of IBRs connected to weak distribution networks and their ability to provide multiple dynamic support services, as well as the technical challenges/requirements associated with a) IBR converter-level control loops (e.g., PLL, current controllers), $b$ ) external system physical features (low system strength, grid resistive feature), and $c$ ) grid-code requirements (e.g., angle jump requirement). While doing so, we also specifically address relevant issues associated with small-signal and large-signal analysis, as we elaborate below.

With regard to small-signal analysis (e.g., up to $1-2 \mathrm{~Hz}$ frequency oscillations following generation/load outage/shedding in conventional power systems [20], which may be even larger in low-inertia future grids [2]), this work investigates the potential benefits in frequency response from IBRs. Moreover, we mathematically discuss how this frequency response may result in voltage violations due to low system strength. In addition, we discuss and study how the physical characteristics of weak distribution grids call for specific design requirements on IBR converter control loops, particularly $d-q$ current control, to ensure their stable operation/dynamic response.

In the case of large-signal voltage disturbances, we mathematically discuss the technical issues with IBR active power control in weak distribution feeders while also highlighting the role of IBR reactive power in their converterlevel stability. To this end, disturbance performance characteristics of converter-based resources in weak distribution grids are proposed which determine the requirements for IBR reactive power support at a minimum active power output during abnormal terminal voltage conditions. Then, we integrate the proposed disturbance performance characteristics into IBR converter-level control loops, in particular active/reactive power reference signal generation strategy, while also taking into account grid-code requirements (e.g., angle jump constraint). Possible IBR active-reactive power interaction due to low system strength/grid-code requirements is discussed as well as its corresponding implications on actual capabilities of IBRs in providing multiple dynamic responses.

The main contributions of the paper are as follows:

- Present, for the first time, the mathematical foundations to study IBR dynamics in weak distribution grids for every possible operating condition;

- Study the potential benefits from providing multiple dynamic support responses from IBRs as well as the challenges/requirements associated with low system strength/ distribution grid with resistive components;

- Propose suitable disturbance performance characteristics for IBRs in weak distribution feeders;

- Assess the impacts of grid-code requirements and of converter-level control instability on the disturbance performance characteristics of IBRs;

- Introduce an IBR reference generation strategy that prioritizes its reactive power at a minimum active power output during abnormal voltage conditions which may cause possible interactions of active-reactive responses.

The paper is organized as follows: Section II presents the small-signal analysis of IBR dynamics in weak distribution grids and its control design requirements. Section III discusses how IBRs should react to large-signal disturbances in weak distribution grids and proposes suitable disturbance performance characteristics. The efficacy of the proposed characteristics and design requirements is evaluated in Section IV. Section V provides the concluding remarks.

\section{IBR DYNAMICS FOLLOWING SMALL-SIGNAL DISTURBANCES IN WEAK DISTRIBUTION GRIDS}

\section{A. IBR d-q Current Dynamics in Weak Distribution Grids}

Most distribution-connected IBRs have a rated capacity in the range from tens of kVA (e.g., $50 \mathrm{kVA}$ ) to a few MVA (e.g., 5 MVA) and are typically equipped with single-stage current-controlled voltage source converters (CC-VSCs). This allows an IBR to handle its active power $(P)$ through $d$-axis current $\left(i_{d}\right)$ control while it manages its reactive power output $(Q)$ via $q$-axis current $\left(i_{q}\right)$ control [21]. Park's transformation (PT) is used to convert the three-phase voltage/current values, which are in the grid synchronous reference frame with $\omega_{s}$ radial frequency, to their $d q$-axis values, which are in the converter reference frame with $\omega_{P L L}$ radial frequency. Also, its inverse ("PT inverse" block in Fig. 1) transforms the $d q$-axis voltage values to its equivalent three-phase values. 


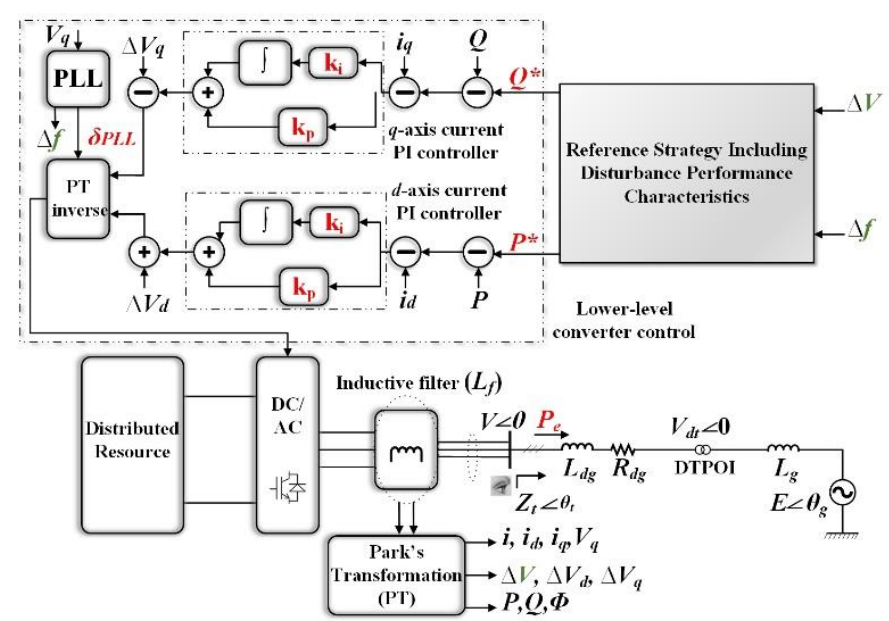

Fig. 1. An IBR connected to a weak distribution grid and equipped with the proposed disturbance performance characteristics.

Consider a converter-based resource connected to a weak distribution grid (Fig. 1) whose transformers and lines are modelled via an equivalent impedance of $Z_{d g}=R_{d g}+\omega_{s} L_{d g}$, where $R_{d g}$ is the grid equivalent resistance and $L_{d g}$ is its equivalent inductance. The upstream transmission grid is also represented via its Thevenin equivalent model, i.e., an ideal voltage source $\left(E \angle \theta_{g}\right)$ in series with an inductance $\left(L_{g}\right)$. If $V_{d t} \angle 0$ is the DTPOI voltage and $V \angle \theta$ the IBR terminal voltage, the dynamic equations of IBR $d-q$ currents are as follows:

$$
\begin{gathered}
L_{d g} \frac{d i_{d}}{d t}=V \cos (\theta)-V_{d t}-R_{d g} i_{d}+\omega_{s} L_{d g} i_{q} \\
L_{d g} \frac{d i_{q}}{d t}=V \sin (\theta)-R_{d g} i_{q}-\omega_{s} L_{d g} i_{d}
\end{gathered}
$$

Let us then assume a small-signal disturbance causes small-signal oscillations in voltage $(V)$, angle $(\theta)$, and $d-q$ currents $\left(i_{d}, i_{q}\right)$ at the IBR terminals. This means $V=V_{0}+\Delta V$, $\theta=\theta_{0}+\Delta \theta, i_{d}=I_{d 0}+\Delta i_{d}$, and $i_{q}=I_{q 0}+\Delta i_{q}$, where $\left(V_{0}\right.$, $\left.\theta_{0}, I_{d 0}, I_{q 0}\right)$ represents the pre-contingency operating point of the system variables at the terminals. It should be noted that such simultaneous changes in different system variables (current, voltage, angle) relate to the low system strength conditions and low short-circuit level at the DTPOI. This makes the following mathematical discussions different from the classical small-signal stability analysis [20]. We apply the above variable changes to (1)-(2), linearize them as $\sin \left(\theta_{0}+\right.$ $\Delta \theta) \approx \sin \left(\theta_{0}\right)+\cos \left(\theta_{0}\right) \Delta \theta$ and $\cos \left(\theta_{0}+\Delta \theta\right) \approx \cos \left(\theta_{0}\right)-$ $\sin \left(\theta_{0}\right) \Delta \theta$, and then write them in Laplace domain as:

$$
\begin{aligned}
& \Delta i_{d}=G_{1}(s) \Delta \theta+G_{2}(s) \Delta V \\
& \Delta i_{q}=G_{3}(s) \Delta \theta+G_{4}(s) \Delta V
\end{aligned}
$$

where

$$
\begin{gathered}
G_{1}(s)=V_{0} \frac{\omega_{s} L_{d g} \cos \left(\theta_{0}\right)-\left(L_{d g} s+R_{d g}\right) \sin \left(\theta_{0}\right)}{\left(L_{d g} s+R_{d g}\right)^{2}+\left(\omega_{s} L_{d g}\right)^{2}} \\
G_{2}(s)=\frac{\omega_{s} L_{d g} \sin \left(\theta_{0}\right)+\left(L_{d g} s+R_{d g}\right) \cos \left(\theta_{0}\right)}{\left(L_{d g} s+R_{d g}\right)^{2}+\left(\omega_{s} L_{d g}\right)^{2}} \\
G_{3}(s)=V_{0} \frac{\omega_{s} L_{d g} \sin \left(\theta_{0}\right)+\left(L_{d g} s+R_{d g}\right) \cos \left(\theta_{0}\right)}{\left(L_{d g} s+R_{d g}\right)^{2}+\left(\omega_{s} L_{d g}\right)^{2}} \\
G_{4}(s)=\frac{-\omega_{s} L_{d g} \cos \left(\theta_{0}\right)+\left(L_{d g} s+R_{d g}\right) \sin \left(\theta_{0}\right)}{\left(L_{d g} s+R_{d g}\right)^{2}+\left(\omega_{s} L_{d g}\right)^{2}}
\end{gathered}
$$

From (3)-(8), it can be appreciated how any change in IBR $d-q$ current outputs $\left(\Delta i_{d}, \Delta i_{q}\right)$, from either IBR converter control adjustments or small-signal system disturbances, can potentially affect both angle and voltage dynamics at the IBR terminal. In particular, angle $(\Delta \theta)$ and voltage $(\Delta V)$ variations are more likely to happen at the same time. This is different from strong grid connections where the most prominent dynamics is system angle/frequency swing following smallsignal disturbances, as discussed in [20]. Furthermore, considering the resistive characteristic of distribution grids $\left(R_{d g} \gg L_{d g}\right), G_{l}(s)$ and $G_{3}(s)$ have double-poles on the negative real axis $\left(Z_{G} \approx \frac{-R_{d g}}{L_{d g}}\right)$ with no zero. Having poles on the lefthand side of the $s$-plane means that the small-signal changes in IBR active/reactive power do not harm/destabilize terminal angle dynamics. However, having no zero means there may be instantaneous, concurrent interactions between IBR $d-q$ current controls (active/reactive power controls) and IBR terminal angle dynamics at weak connections. Such instantaneous dynamic phenomena normally do not occur at strong distribution connections.

\section{B. Possible Voltage Variations Following IBR Frequency Response}

It is of interest to study possible challenges in frequency response provision from IBRs in weak distribution grids.

Let us consider the most common practice in IBRs where active power output is governed via $d$-axis current controller as a response to a given frequency disturbance $(\Delta f)$ [21]. The $i_{d}$ impact on IBR terminal voltage $(V)$ can be captured via $G_{2}(s)$ while $L_{d g}$ is replaced by $L_{d g}+L_{g}$ to include the external grid inductance. If $s \rightarrow 0$ in $G_{l}(s)$, the IBR steady-state terminal voltage change $\left(\Delta V_{s s}\right)$ following a given $i_{d}$ change $\left(\Delta i_{d, s s}\right)$ can be calculated from (9):

$$
\Delta V_{s S}=\frac{\omega_{s}\left(L_{d g}+L_{g}\right)}{\sin \left(\theta_{0}\right)} \Delta i_{d, s s} \approx \frac{\omega_{s} L_{g}}{\sin \left(\theta_{0}\right)} \Delta i_{d, s s}
$$

Considering the Thevenin equivalent voltage $E=1$ p.u. and IBR nominal power $I^{r}=1$ p.u., the short-circuit ratio at the DTPOI $\left(S C R_{D T P O I}\right)$ equals to $\frac{1}{\omega_{s} L_{g}}$, where $L_{g}$ is the external grid inductance. Eq. (9) can then be written as follows:

$$
\Delta V_{S S} \approx \frac{1}{S C R_{D T P O I} \sin \left(\theta_{0}\right)} \Delta i_{d, S S}
$$

Considering weak distribution grids with very low $S C R_{D T P O I}$ values (e.g., $S C R_{D T P O I}<3$ is considered as a weak grid connection in Australia [22]), Eq. (10) indicates that the IBR active power change in the form of $d$-axis current change is more likely to cause voltage variations at the DTPOI. Such voltage deviations may, in turn, prevent further frequency support from DERs in weak distribution grids. This interaction of IBR frequency support with the DTPOI voltage dynamics must be considered in security and market analysis to capture the actual IBR frequency response capabilities.

Let us now discuss the less frequent case in which IBR controls its active power via $q$-axis current controller. Using $G_{4}(s)$ in (8), a similar approach as above can be taken to calculate the voltage change following a given $\Delta i_{q}$, as in (11).

$$
\Delta V_{S S} \approx \frac{1}{S C R_{D T P O I} \cos \left(\theta_{0}\right)} \Delta i_{q, S S}
$$

Eq. (11) indicates again that IBR active power response to 
$\Delta f$ may lead to voltage variations at weak grid connections; this may, in turn, prevent provision of further frequency support from IBRs. We can therefore conclude that such behavior is irrespective of the IBR converter-level control strategy ( $d-q$ current controls), but it rather relates to the physical features of the external system $\left(S C R_{D T P O I}\right)$. Finally, it follows from both (10) and (11) that the potential frequency/voltage interaction is also associated with the precontingency value of the IBR terminal voltage angle $\left(\theta_{0}\right)$. This in turn depends on the pre-contingency load and generation operating profiles.

\section{IBR Control Design Requirements at Weak Distribution Grid Connections}

Considering the focus in this section on small-signal analysis, we can linearize the IBR dynamic equations around an equilibrium point to obtain its state-space model as below:

$$
\begin{gathered}
\left(\begin{array}{c}
\frac{d i_{d}}{d t} \\
\frac{d i_{q}}{d t}
\end{array}\right)=\left(\begin{array}{cc}
0 & -k_{i} \\
\frac{1}{L_{t}} & \frac{-\left(R_{d g}+k_{p}\right)}{L_{t}}
\end{array}\right)\left(\begin{array}{l}
i_{d} \\
i_{q}
\end{array}\right) \\
+\left(\begin{array}{l}
k_{i} \\
\frac{k_{p}}{L_{t}}
\end{array}\right)\left(\begin{array}{l}
i_{d}^{r e f} \\
i_{q}^{r e f}
\end{array}\right)
\end{gathered}
$$

where $\left(k_{i}, k_{p}\right)$ are the coefficients of the $d-q$ current proportional-integral (PI) controllers (see Fig. 1), and $L_{t}=L_{f}+L_{d g}+L_{g}$.

The eigenvalues of the state matrix in (12) are:

$$
\lambda_{1,2}=\frac{\frac{-\left(R_{d g}+k_{p}\right)}{L_{t}} \pm \sqrt{\left(\frac{R_{d g}+k_{p}}{L_{t}}\right)^{2}-\frac{4 k_{i}}{L_{t}}}}{2}
$$

The IBR $d-q$ current control is stable if the real part of $\lambda_{1,2}$ is in the left hand-side of the $s$-plane. This holds if:

$$
k_{p}>\left(-R_{d g}\right), k_{i}>0
$$

It is therefore important to notice that the external system physical characteristic $\left(R_{d g}\right)$ even affects the IBR converter control parameters too. Further, the smaller is the $k_{p}$ value, the faster the IBR response would be. A large value of $k_{i}$ could also reduce the quasi steady-state error of IBR response. The relationship in (14) represents a design requirement that guarantees a stable, desired frequency control from IBRs in weak distribution grids, unless the DTPOI voltage is violated (see Section III for that case). Finally, the proposed constraints in (14) indicate that fine-tuning of the $d$ - $q$ current controllers' coefficients can potentially be a solution to mitigate smallsignal oscillations at weak grid connections. While this is consistent with recent general industry suggestions to mitigate small-signal oscillations in weak grids [23], we provide here for the first time a mathematical formulation of the required conditions. For example, the current control tuning has recently been identified by the Australian system operator as a potential mitigation solution for small-signal oscillations in weak grids [23]. What suggested by the system operator is general through and no specific guidelines and design requirements have been proposed thus far.

\section{IBR DYNAMICS FOLLOWING LARGE-SIGNAL DISTURBANCES IN WEAK DISTRIBUTION GRIDS}

\section{A. IBR $d-q$ Current Dynamics Following Large-Signal Voltage Disturbances}

A large-signal voltage disturbance results in significant changes in system voltages and currents (torques), i.e., $V=V_{0}+$ $\Delta V, i_{d}=I_{d 0}+\Delta i_{d}{ }^{\prime}$, and $i_{q}=I_{q 0}+\Delta i_{q}{ }^{\prime}$. Using (1)-(2) and Laplace transformation, the IBR $d-q$ current changes can then be expressed as per (15)-(16).

$$
\begin{gathered}
\Delta i_{d}{ }^{\prime}=\frac{\omega_{s} L_{t} \sin \left(\theta_{0}\right)+\left(L_{t} s+R_{d g}\right) \cos \left(\theta_{0}\right)}{\left(L_{t} s+R_{d g}\right)^{2}+\left(\omega_{s} L_{t}\right)^{2}} \cdot \Delta V \\
\Delta i_{q}{ }^{\prime}=\frac{-\omega_{s} L_{t} \cos \left(\theta_{0}\right)+\left(L_{t} s+R_{d g}\right) \sin \left(\theta_{0}\right)}{\left(L_{t} s+R_{d g}\right)^{2}+\left(\omega_{s} L_{t}\right)^{2}} \cdot \Delta V
\end{gathered}
$$

\section{B. Ineffective IBR P-V Control in Weak Distribution Grids}

The synchronizing power $P_{e}$ between DER terminals and the external grid (see Fig. 1) can be formulated as follows:

$$
P_{e}=V\left(i_{d} \cos \left(\delta_{P L L}\right)-i_{q} \sin \left(\delta_{P L L}\right)\right)
$$

$P_{e}$ should be ideally equal to IBR active power output $(P)$, i.e., $P_{e}=P$. However, there are two main contributing factors to $P_{e}$ being different from $P: i$ ) IBR $d$ - $q$ current adjustments, and ii) large-signal disturbances. In both cases, the synchronizing power change $\left(\Delta P_{e}\right)$ can be obtained from (18):

$$
\Delta P_{e}=\left[\begin{array}{c}
I_{d 0} \\
I_{q 0}
\end{array}\right]^{T} \cdot\left[\begin{array}{l}
\Delta V_{d} \\
\Delta V_{q}
\end{array}\right]+\left[\begin{array}{c}
V_{d 0} \\
V_{q 0}
\end{array}\right]^{T} \cdot\left[\begin{array}{c}
\Delta i_{d}{ }^{\prime} \\
\Delta i_{q}{ }^{\prime}
\end{array}\right]
$$

where $\left(I_{d 0}, I_{q 0}, V_{d 0}, V_{q 0}\right)$ are the pre-contingency values of IBR terminal current and voltage in $d-q$ coordinates. Let us first assume IBR does not experience converter-level instability, in particular PLL instability, following a given large-signal disturbance. Under this assumption, IBR is able to control its $d-q$ currents properly, which results in stable performance in active/reactive power control. Considering a pre-contingency value of the IBR terminal angle $\theta=\theta_{0}$ and replacing (15)-(16) into (18), the change in $P_{e}$ (resp. $P$ ) with respect to a given $\Delta V$ can be formulated as follows:

$$
\Delta P_{e}=\frac{\left(L_{t} s+R_{d g}\right)\left(2 V_{0}-E_{0} \cos \left(\theta_{0}\right)\right)-E_{0} \omega_{s} L_{t} \sin \left(\theta_{0}\right)}{\left(L_{t} s+R_{d g}\right)^{2}+\left(\omega_{s} L_{t}\right)^{2}} \Delta V
$$

Eq. (19) indicates that change in IBR active power can potentially affect voltage dynamics at the IBR terminal and vice versa. However, the transfer function in (19) has a zero in $s=\frac{E_{0} \omega \sin \left(\theta_{0}\right)}{2 V_{0}-E_{0} \cos \left(\theta_{0}\right)}-\frac{R_{d g}}{L_{t}}$. From control system theory principles, zeros add a certain time delay between system input and its output [20]. Interestingly, the zero in (19) is associated with the external grid features and does not depend on IBR converter control. Most importantly, the associated delay can potentially prevent effective voltage control through IBR active power control ( $P-V$ control). This comes from the fact that the IBR terminal voltage $(V)$ has very fast dynamics due to low system strength. Even though the IBR is able to control its active power as a response to $\Delta V$, such $P-V$ control is not effective since it is a response to a certain voltage deviation which may no longer exist! This is in stark contrast to the traditional $P-V$ control concept applied in distribution grids [24]. In particular, this ineffectiveness in $P-V$ control 
emerges from $i$ ) external system features (associated with $s=$ $\left.\frac{E_{0} \omega \sin \left(\theta_{0}\right)}{2 V_{0}-E_{0} \cos \left(\theta_{0}\right)}-\frac{R_{d g}}{L_{t}}\right)$, and $\left.i i\right)$ high voltage sensitivity $(\Delta V)$ due to low system strength. In addition, this ineffective $P-V$ control may, in turn, worsen the terminal voltage variations which may eventually result in IBR converter instability and possible disconnection due to loss of synchronism. Finally, the mentioned IBR $P-V$ control issue is more likely to happen in IBRs located at the end of a distribution feeder. In fact, since DERs connected to the end of a feeder see a higher equivalent resistance $\left(R_{d g}\right)$, the mentioned zero is closer to the imaginary axis $\left(\frac{R_{d g}}{L_{t}}\right.$ is larger and therefore $\left(s=\frac{E_{0} \omega \sin \left(\theta_{0}\right)}{2 V_{0}-E_{0} \cos \left(\theta_{0}\right)}-\frac{R_{d g}}{L_{t}}\right)$ is smaller), thereby resulting in longer delays between voltage inputs and IBR active power response.

\section{Impacts of IBR Active/Reactive Power Control on its PLL Stability/DER Synchronism}

Let us now assume IBR may face converter instability following a large-signal disturbance. The IBR converter stability highly depends on how accurate its PLL performance is in estimating the terminal voltage angle $(\theta)$. The IBR PLL should ideally estimate $\theta$ by keeping the $q$-axis terminal voltage $\left(V_{q}\right)$ equal to zero, i.e., if $V_{q}=0$ then $\delta_{P L L}=\theta$ (see Fig. 2(a)). The PLL PI controller in the phase-locking strategy, illustrated in Fig. 2(b), regulates $V_{q}$. The estimated PLL angle at any given time $t$ can be formulated as per (20):

$$
\begin{array}{r}
\delta_{P L L}(t)=\int_{0}^{t}\left[\omega_{s}+K_{P}^{P L L} \cdot V_{q}(t)+\right. \\
\left.\int_{0}^{t}\left(K_{I}^{P L L} \cdot V_{q}(t)\right) d t\right] d t
\end{array}
$$

where $\left(K_{P}^{P L L}, K_{I}^{P L L}\right)$ are the coefficients of the PLL PI controller. It is then important to understand what contributes to $\delta_{P L L} \neq \theta$ and its mitigation strategies. From Fig. 2(a), the $q$ axis terminal voltage can be formulated as follows:

$$
V_{q}=E \cdot \sin \left(\theta_{g}-\delta_{P L L}\right)+i \cdot Z_{t} \cdot \sin \left(\theta_{t}+\phi\right)
$$

where $i>0$ is the IBR output current, $Z_{t} \angle \theta_{t}$ is the impedance seen from the IBR terminal (see Fig. 1), and $\phi$ is the angle between IBR voltage and its current. Considering $\varepsilon=\delta_{P L L}-$ $\theta_{g}$ and $\theta_{g}=\int \omega_{s} d t$, replacing (21) into (20) results in (22), which describes the IBR PLL dynamics:

$$
\begin{gathered}
\varepsilon=\int\left(k_{P}^{P L L}+k_{I}^{P L L} \int\right)(-E \cdot \sin (\varepsilon)+ \\
\left.i . Z_{t} \cdot \sin \left(\theta_{t}+\phi\right)\right)
\end{gathered}
$$

(a)

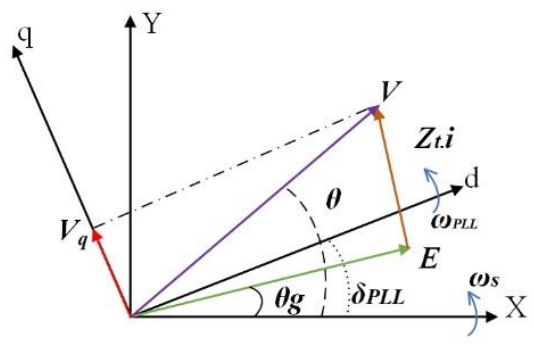

(b)

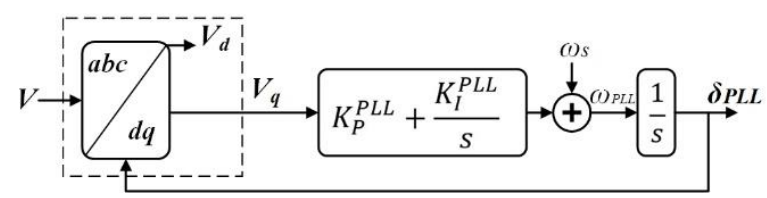

Fig. 2. (a) Vector diagram illustrating angles/voltages in PLL-synchronous reference frame, (b) PLL model.

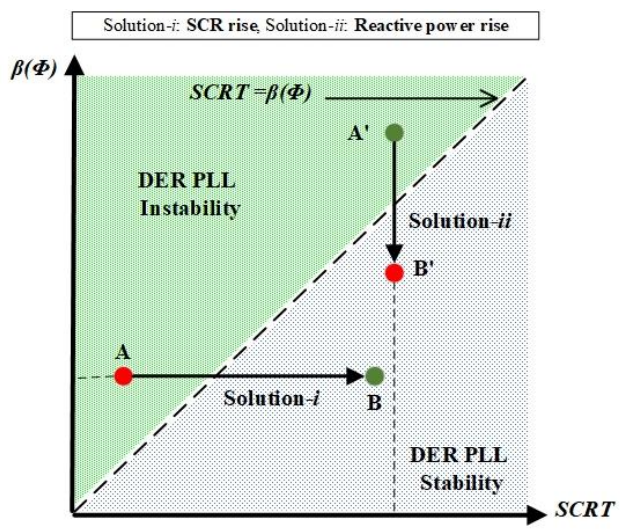

Fig. 3. IBR PLL stability via solution- $i$ : $S C R_{T}$ rise, solution-ii: reactive power control.

Assuming the IBR rated current $I_{D E R}^{r}=1$ p.u., the shortcircuit ratio at the IBR terminals is equal to $S C R_{T}=\frac{E}{z_{t}}$. Eq. (22) can then be written as:

$$
\begin{array}{r}
\varepsilon=\int\left[( k _ { P } ^ { P L L } + k _ { I } ^ { P L L } \int ) \left(-S C R_{T} \cdot Z_{t} \cdot \sin (\varepsilon)\right.\right. \\
\left.\left.+i \cdot Z_{t} \cdot \sin \left(\theta_{t}+\phi\right)\right)\right]
\end{array}
$$

If $\quad\left(-S C R_{T} \cdot Z_{t} \cdot \sin (\varepsilon)+i \cdot Z_{t} \cdot \sin \left(\theta_{t}+\phi\right)>0\right)$, the difference between the IBR PLL estimated angle $\left(\delta_{P L L}\right)$ and the grid angle $\left(\theta_{g}\right)$ is prone to increase following a given contingency. Therefore, the IBR PLL could not find an equilibrium point. The PLL may fail to regulate $V_{q}$ and estimate the terminal angle $(\theta)$, which may eventually lead to PLL instability and IBR loss of synchronism. Based on this, PLL stability can be guaranteed following large-signal disturbances if the following constraint holds:

$$
S C R_{T}>\frac{i \cdot \sin \left(\theta_{t}+\phi\right)}{\sin (\varepsilon)}=\beta(\phi)
$$

The constraint proposed in (24) suggests the following solutions to enhance IBR PLL/converter stability:

i) The first and most common solution is to increase $S C R_{T}$ at the IBR POI (Fig. 3, solution-i), e.g., via synchronous condenser installation at the IBR terminals, upstream transmission grid enhancement, etc. This is consistent with industry developments [25];

ii) Since $\theta_{t} \simeq \frac{\pi}{2}$, a second possible solution is to decrease $\beta(\phi)$ via increasing $\phi$, which corresponds to increasing the IBR reactive power exchange with the external system (Fig. 3, solution-ii). The proposed constraint thus clearly highlights the importance of IBR reactive power for its stable operation in weak distribution grids. This solution is also in line with recent industry suggestions [22] for IBR converter controls. It is to be noted, also, that such reactive power control would constrain active power output due to converter thermal limits, thus limiting the IBR frequency response capability too.

iii) A third possible solution is to curtail IBR output current $(i>0)$ to reduce $\beta(\phi)$. This is somehow consistent with suggestions from inverter manufactures (e.g., [26]), who advise for IBR not to inject current into the grid (i.e., $i=0$ ) following severe voltage disturbances. However, the 
manufactures' advice may not be feasible in the context of this study since there is no guarantee that an equilibrium point can be found for voltages in distribution feeders when IBRs transition to "temporary cessation mode", as discussed in [27].

Finally, the proposed constraint in (24) is obtained without linearizing the PLL dynamics. This is then consistent with the principles of large-signal analysis where it may not be legitimate to employ dynamic model linearization [20].

\section{Proposed Disturbance Performance Characteristics of IBRs in Weak Distribution Grids}

IBR active power increase following large-signal voltage disturbances is not helpful for both system voltage/frequency control (Section III-B) and IBR PLL/converter stability (Section-III-C). In light of the earlier discussions, this work therefore proposes the IBR disturbance performance characteristics shown in Fig. 4, which define how IBRs should behave in the following system conditions:

Normal case: if IBR voltage terminal is within normal operating band $\left(\Delta V<V_{I}\right)$ and there is no frequency disturbance $\left(|\Delta f|<F_{D}\right)$, IBRs should operate at a desired/pre-defined $P$ while adjusting their $Q$ to comply with power factor grid-code requirements, so-called voltage control response [3]. The IBR voltage control response is adjusted according to $P-Q$ capability diagrams [3] as per Fig. 4(c).

Frequency disturbance case: when there is a frequency disturbance with no voltage violation $\left(\Delta V<V_{l},|\Delta f|>F_{D}\right)$, IBRs should give priority to active power support as a response to $\Delta f$, according to Fig. 4(d), and also handle their terminal voltage via $Q$ control if there is enough converter capacity. This reactive power control should be adjusted based on IBR $P-Q$ capability diagrams (Fig. 4(c)). Also, the IBR frequency control response depends on the active powerfrequency droop coefficient, which is typically determined according to the frequency control requirements defined by system operators [6]. The IBR frequency response however should not cause voltage violations, as per Section-II-B.

Concurrent $f-V$ disturbance case: when both voltage and frequency are beyond their normal operating bands $\left(\Delta V>V_{l}\right.$, $\left.|\Delta f|>F_{D}\right)$, IBRs should exchange reactive power with the grid to the greatest possible extent to 1) support their own converter stability (Section III-C), and 2) possibly mitigate voltage excursions emerged from the fault. Since the distribution grid may have a large resistive component, the consequent voltage improvement would have to rely on a significant volume of reactive power provision. This would depend on the volume of IBRs as well as their voltage-reactive power droop value (Fig. 4(a)). In addition, the IBR active power should immediately drop to a minimum level $P_{\min }$ (Fig. 4(b)), which should be determined by system operators. This may, in turn, prevent frequency response from the IBR.

Angle jump case: considering the principles of droop control in resistive networks [24], the IBR reactive power exchange following a large-signal disturbance can potentially result in angle changes, which may violate the angle jump requirement defined by system operators. This is captured in Fig. 4(a) where IBRs should stop further reactive power support to the system.

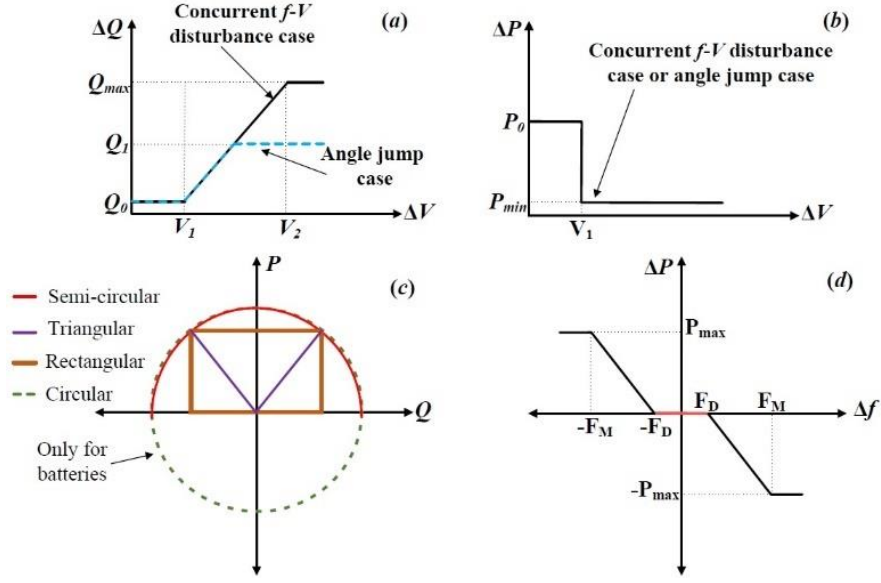

Fig. 4. Proposed disturbance performance characteristics for IBRs at weak distribution network connections.

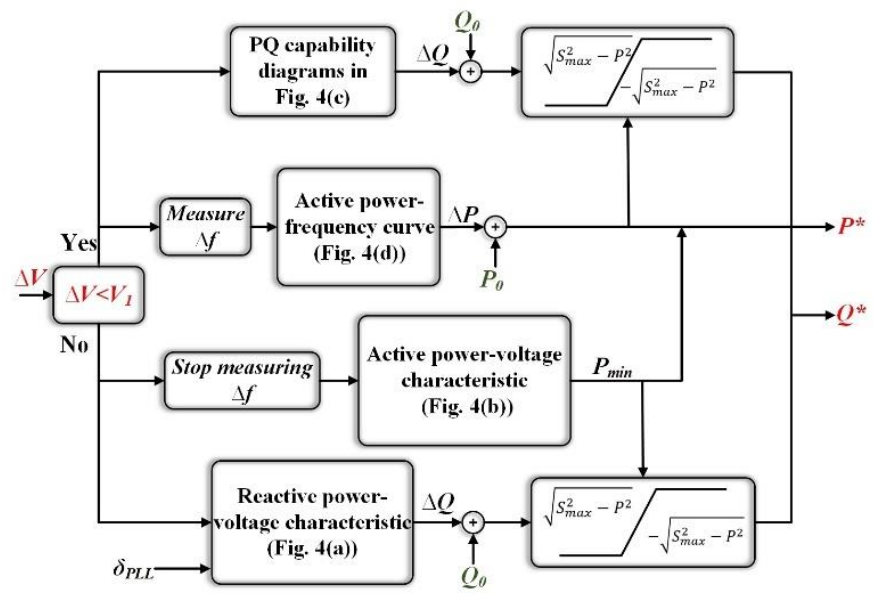

Fig. 5. Proposed voltage-priority reference generation strategy for IBRs in weak distribution grids including the disturbance performance characteristics.

In the above cases, the IBR active-reactive power outputs must remain within its converter power transfer limit $\left(S_{\max }\right)$. Finally, the proposed characteristics are then integrated into the IBR $P-Q$ reference generation strategy (Fig. 5) to generate inputs $\left(P^{*}, Q^{*}\right)$ for the converter control loops. The proposed voltage-priority strategy (Fig. 5) and its associated disturbance performance characteristics (Fig. 4) only require three input signals to preserve stable converter control operation and IBR synchronism: 1) the IBR terminal voltage to calculate possible voltage variations $(\Delta V)$, which can typically be obtained from a voltage measurement device at the IBR terminal; 2) the system frequency to compute possible frequency variations $(\Delta f)$, and 3) the PLL estimated angle $\left(\delta_{P L L}\right)$. Both $\delta_{P L L}$ and $\Delta f$ can be obtained from the embedded phase-locking strategy in the IBR converter control, as per Fig. 1. In particular, none of the mentioned signals require a steady-state condition in the external system. In this work, in fact, IBRs do not have any knowledge/information about the system beyond their terminals and IBR operation and responses are only based on parameters measured at their terminals. This is also consistent with typical IBR operation where the IBR terminal parameters are measured and used for converter control purposes [21], [23]. 


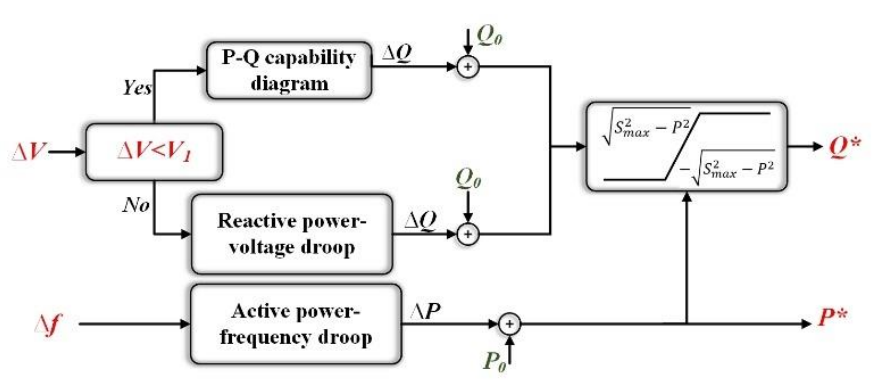

Fig. 6. Frequency-priority $P-Q$ reference generation strategy for IBRs.

To be noted is that the voltage sampling frequency should not be less than the system rated frequency $\left(f_{s}\right)$, in order to ensure adequate tracking of the IBR terminal voltage magnitude dynamics. This would necessitate a voltage measurement delay of less than $T_{s}=\frac{1}{f_{s}}=\frac{2 \pi}{\omega_{s}}$. In addition, the PLL sampling frequency should be several times higher than the rated grid frequency to ensure adequate tracking of the IBR terminal angle dynamics, which is essential for stable converter control operation and IBR synchronism in weak grids.

While the proposed voltage-priority approach can potentially prevent IBR lower-level converter control instability (see Fig. 1) following system contingencies, it cannot be helpful in the case when the lower-level converter control is originally unstable due to inappropriate adjustment of its controllers' coefficients. Therefore, a certain level of control tuning should be in place to ensure the IBR lowerlevel control capability in tracking the generated reference signals $\left(P^{*}, Q^{*}\right)$. Further, the proposed voltage-priority strategy can contribute to mitigating possible negative interactions among multiple IBRs by reducing the active power injection from IBRs to a minimum level $P_{\min }$ (Fig. 4(b)) when their terminal voltage were to be in abnormal conditions $\left(\Delta V>V_{l}\right)$. In Section IV, the performance of the proposed strategy will be evaluated and compared to the case where IBR prioritizes its active power support over its reactive power support in every voltage condition (Fig. 6).

We would like to emphasize that there is no specific value assumed for the $S C R_{D T P O I}$ value in the above discussions. This means that the presented mathematical discussions, the disturbance performance characteristics (Fig. 4), and the voltage-priority approach (Fig. 5) hold for IBR operation and dynamics in any distribution grid, including weak ones with a low $S C R_{D T P O I}$ value, e.g., in the order or 1 or 2 . Finally, there is no assumption on the rate of change of IBR $d q$-axis currents and their associated magnitudes in the above discussions. Our mathematical analysis thus indicates that the IBR instability may happen in weak distribution grids even before IBR current/ramp limiters operate.

\section{CASE STUdies}

This section includes three case studies:

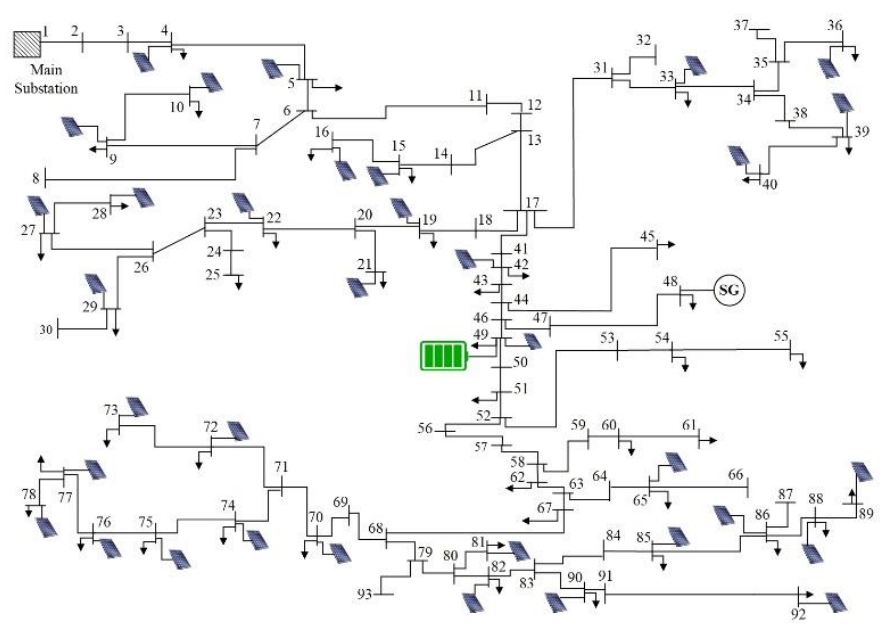

Fig. 7. A real Australian PV-rich distribution feeder with a low SCR value at the main substation $\left(S C R_{D T P O} \approx 2\right)$.

Case- $i$ demonstrates the importance of IBR $d-q$ current control tuning and the design requirements proposed in (14) to guarantee a stable, desirable response in weak distribution grids. In this regard, we discuss how IBR location, particularly the equivalent resistance $\left(R_{d g}\right)$ seen by the IBR, can potentially impact on the IBR controllers' coefficients.

Case-ii illustrates IBR capabilities in simultaneous active/reactive power response following a given frequency disturbance. The impact of frequency support from IBRs on system voltages at weak grid connections is also discussed.

Case-iii studies how IBRs should operate following largesignal voltage disturbances, also emphasizing the importance of the proposed disturbance performance characteristics (Fig. 4) and the associated voltage-priority reference generation strategy (Fig. 5).

The case studies are presented for a real Australian MV distribution feeder (Fig. 7) connected to a weak part of the grid with $S C R_{D T P O I}$ around 2. This is a $22 \mathrm{kV}$ distribution feeder with 93 nodes which serves a total of 3136 customers out of which 695 customers have PV units with an aggregate capacity of $2.3 \mathrm{MW}$. In addition, the feeder active power load is $12.5 \mathrm{MW}$ while we assume 0.9 power factor for load buses. This feeder also includes eight identical 1.1 MVA synchronous generators connected to bus 48. A 5 MW battery, connected to the feeder via a step-up $11 \mathrm{kV} / 22 \mathrm{kV}$ transformer, is studied here in terms of its dynamic responses from different locations. However, our discussions are not limited to a specific IBR type and can be applied to other converter-based resources (e.g., PV). The model is built in MATLAB/SIMULINK. PV and battery models and parameters are taken from our previous works [4], [6].

The following simulation results and IBR responses are obtained by detailed dynamic modelling of the weak feeder under study, i.e., dynamic modelling of distribution lines and transformers, PV units, battery, as well as the upstream transmission grid. The test system also includes multiple IBRs equipped with the proposed voltage-priority strategy (Fig. 5). 

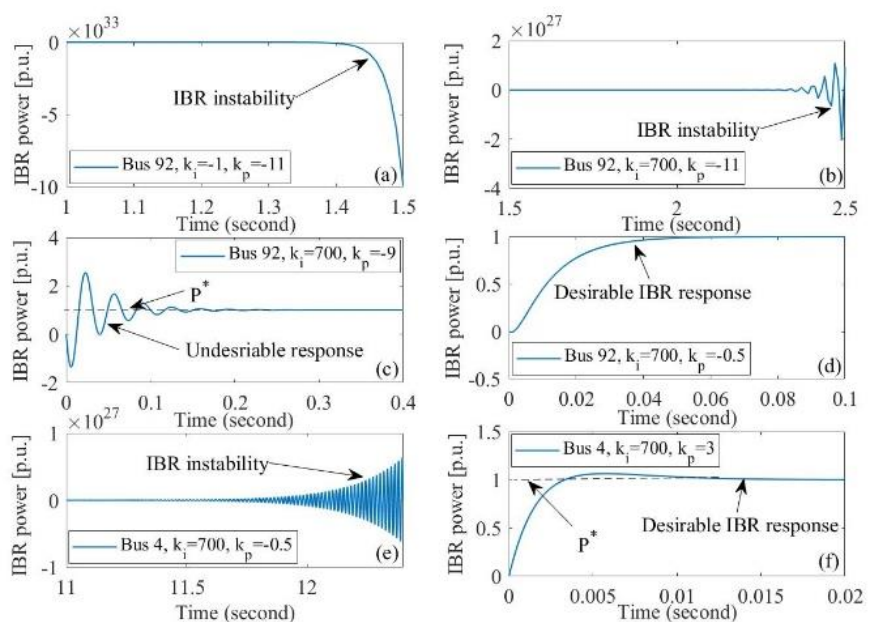

Fig. 8. IBR dynamic behavior to a step-change in its active power reference signal considering different locations and $d-q$ current controllers' coefficients.

\section{A. Case-i: Impacts of Feeder Physical Features on IBR Response Stability and Converter Control Design}

Two different locations for the $5 \mathrm{MW}$ battery are assumed in this section: 1) bus 92 at the feeder end, and 2) bus 4 at the beginning of the feeder. The equivalent resistances $\left(R_{d g}\right)$ seen by the IBR from bus 92 and bus 4 are around $10 \Omega$ and 0.45 $\Omega$, respectively. The battery dynamic response to a stepchange in its active power reference signal $\left(P^{*}\right)$ is shown in Fig. 8 for different values of $d$ - $q$ controllers' coefficients.

Considering IBR installation at bus 92, we first selected IBR controllers' coefficients without explicitly considering the physical features of the feeder $\left(R_{d g}\right)$. To do so, we have employed a standard design approach based on the closedloop transfer functions of IBR $d q$-axis currents. As elaborated in [21], the controllers' coefficients depend on two degrees of freedom, which should be determined by the designer to achieve some specific response characteristics, e.g., settling time and response damping. Assuming $k_{i}=-1$ and $k_{p}=-11$, Fig. 8 (a) shows that the battery could not track $P^{*}$ properly and it faces instability after 1.4 seconds. From (14), we then modified $k_{i}$ so that $k_{i}>0\left(k_{i}=700\right)$ and keeping $k_{p}$ the same. Such large $k_{i}$ value was chosen to mitigate quasi steady-state error in the IBR response. However, Fig. 8(b) shows that $k_{i}$ modification is not enough to stabilize the response. Then, the IBR $d-q$ controllers' coefficients were adjusted so that the proposed requirements in (14) were satisfied (i.e., $k_{i}=700$ and $\left.k_{p}=-9>R_{d g}=-10\right)$. Such coefficient adjustment led to IBR stable dynamic behavior (Fig. 8(c)). However, the response would not be compliant with the grid-code requirements in Australia due to a settling time equal to $100 \mathrm{~ms}$, which is higher than $60 \mathrm{~ms}$ recommended by the Australian system operator [28]. Finally, further increase in $k_{p}\left(k_{p}=-0.5, k_{i}=700\right)$, as per Section II-C, resulted in a desired, stable dynamic response (Fig. 8(d)).

However, the same controllers' coefficients $\left(k_{p}=-0.5\right.$, $k_{i}=700$ ) could not stabilize IBR response for connection at bus 4, as shown in Fig. 8(e). This is because the equivalent resistance seen by the IBR from bus 4 is different from the one seen from bus 92 . This affects the IBR state-space model as per (12) and its corresponding eigenvalues as from (13).
Hence, we then modified $k_{p}$ value further, setting it to $k_{p}=3$. This finally led, also for bus 4 , to IBR response stability and compliance with the desired performance, with response settling time of $10 \mathrm{~ms}$ (Fig. 8(f)). Two important points can thus be concluded: 1) IBR current control tuning based on our proposed design requirements in (14) is an effective way to achieve a stable desired dynamic behavior from IBRs in weak distribution grids. 2) The physical features of the external distribution feeder, in particular the equivalent resistance seen by the IBR, must be considered in IBR converter control design. This can limit the risk of IBRs failing to provide frequency response in distribution grids.

\section{B. Case-ii: Simultaneous Dynamic Responses from IBRs in Weak Distribution Grids}

The feeder under study is then integrated into a modified 14-generator national electricity market (NEM) dynamic model [29] with 50\% renewables, as from the 2030 stepchange scenario in Australia [30-31], to simulate potential frequency/voltage dynamics and multiple simultaneous responses. The system contingency is the largest generation trip, with the capacity of $667 \mathrm{MW}$, at $t=0.4 \mathrm{~s}$. Two scenarios are then considered: Scenario-1) No response provided from the distribution feeder, and Scenario-2) multiple dynamic responses provided from the $5 \mathrm{MW}$ battery, connected to bus 49, and equipped with the proposed strategy in Fig. 5.

In Scenario-1, the largest generation outage results in a significant frequency drop down to $49.55 \mathrm{~Hz}$ (Fig. 9). The system frequency is then stabilized after 7 seconds at quasi steady-state frequency of $49.62 \mathrm{~Hz}$ (Fig. 9) thanks to frequency response from synchronous units in the upstream transmission grid. This contingency also led to small-signal variations in the battery terminal voltage (Fig. 10, Scenario-1).

In Scenario-2, the $5 \mathrm{MW}$ battery delivers simultaneous active/reactive responses to the occurred frequency/voltage disturbances. Fig. 11(a) shows how battery delivers around 4.7 MW frequency response to the system as well as 1 MVar reactive power (Fig. 11(b)) as a voltage control response that mitigates the voltage deviation right after the contingency. The battery frequency response, in turn, resulted in voltage violations at its terminals and took the voltage in each phase above $6.9 \mathrm{kV}$ (Fig. 10). This undesirable dynamic phenomenon relates to the low strength of the distribution feeder. However, the battery then handled this undesirable voltage phenomenon later by sustaining $600 \mathrm{kVar}$ reactive power support to the system (Fig. 11(b)), which led to its terminal voltage stability (Fig. 10, Scenario-2).

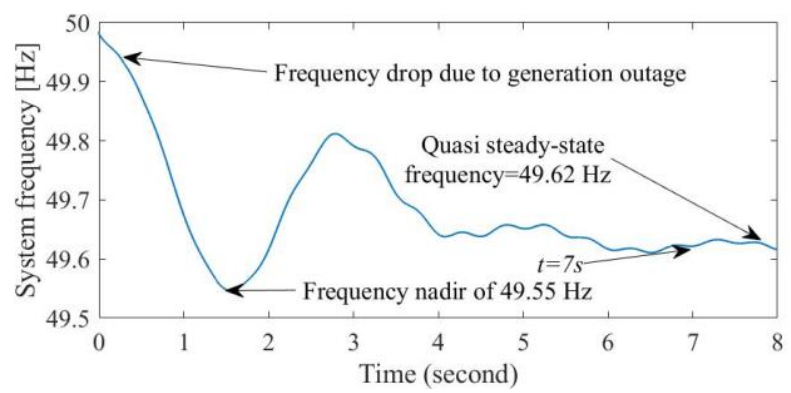

Fig. 9. System frequency excursions following the largest generation outage while there is no response from the battery. 


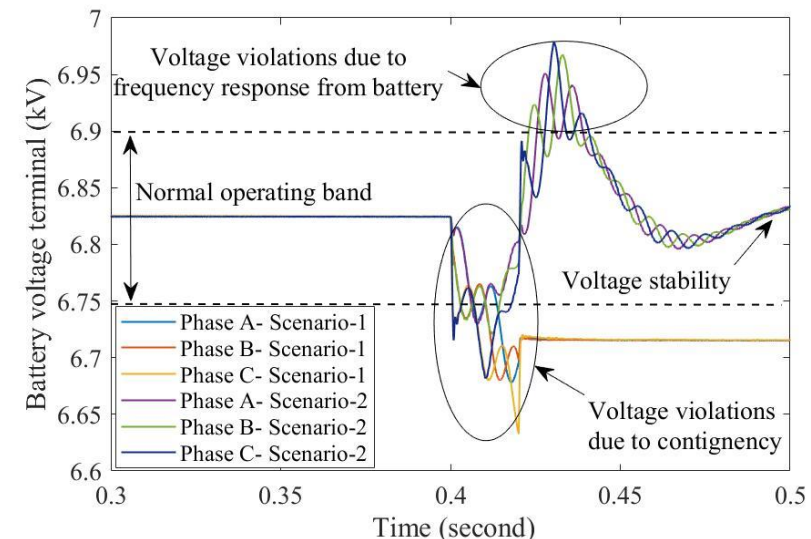

Fig. 10. Voltage dynamics at the battery terminal in two mentioned scenarios while demonstrating voltage violations following its frequency support.
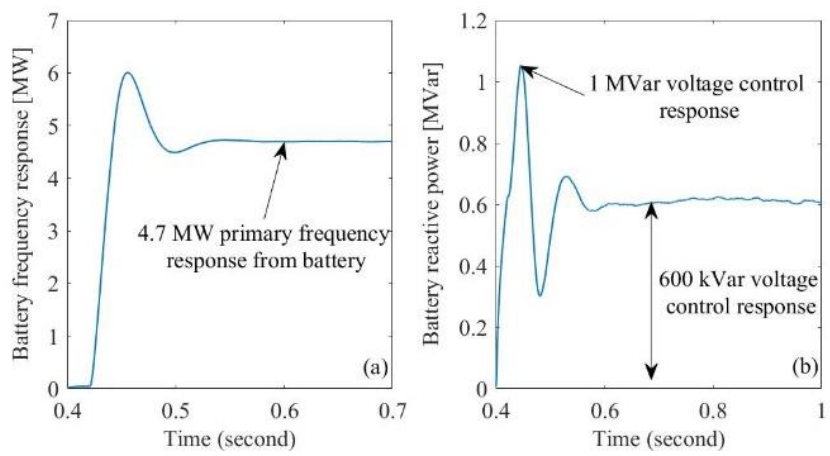

Fig. 11. Simultaneous dynamic responses from $5 \mathrm{MW}$ battery in Scenario-2: (a) frequency response (b) voltage control response.

The simulation results thus indicate: 1) IBRs can provide multiple simultaneous dynamic responses even at weak distribution connections, 2) the efficacy of the proposed disturbance performance characteristics in utilizing IBRs for multiple dynamic supports, and 3) possible voltage violations following IBR frequency supports in weak distribution grids.

\section{Case-iii: Importance of Proposed Disturbance Performance Characteristics}

We apply a phase-to-phase-to-ground short-circuit at the main substation at $t=0.4 \mathrm{~s}$ which lasts for three frequency cycles. The voltage and frequency dynamics are shown in Fig. 12 and Fig. 13, respectively. Such large-signal disturbance results in 57\% voltage drop at the battery terminal (Fig. 12) along with the frequency excursions down to $49.5 \mathrm{~Hz}$ (Fig. 13). Now, let us study the following cases:

- Case-1: $5 \mathrm{MW}$ battery equipped with the proposed frequency-priority reference strategy (Fig. 6);

- Case-2: 5 MW battery equipped with the proposed voltage-priority reference strategy (Fig. 5) which includes the proposed disturbance performance characteristics.

In Case-1, the battery gives the priority to active power control in order to participate in mitigating frequency oscillations. However, the active power rise affected converter-level stability, as per Eq. (24), and led to severe battery active power oscillations during the fault (Fig. 13). Such oscillations might have resulted in battery disconnection if loss of synchronism protection schemes were modelled.

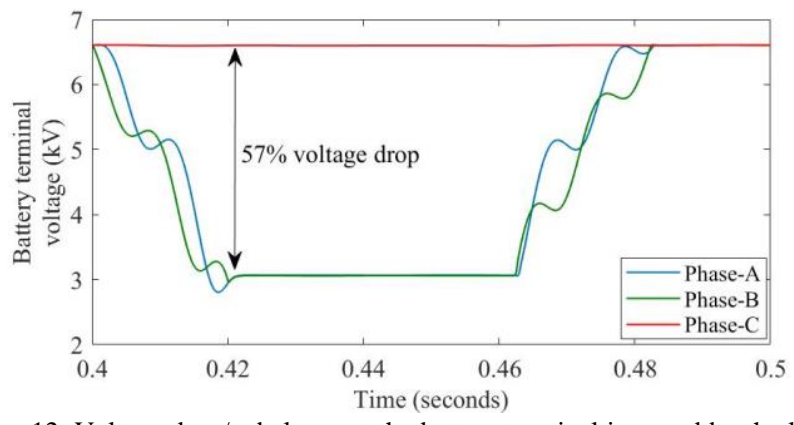

Fig. 12. Voltage drop/unbalance at the battery terminal imposed by the largesignal fault occurred at the DTPOI.

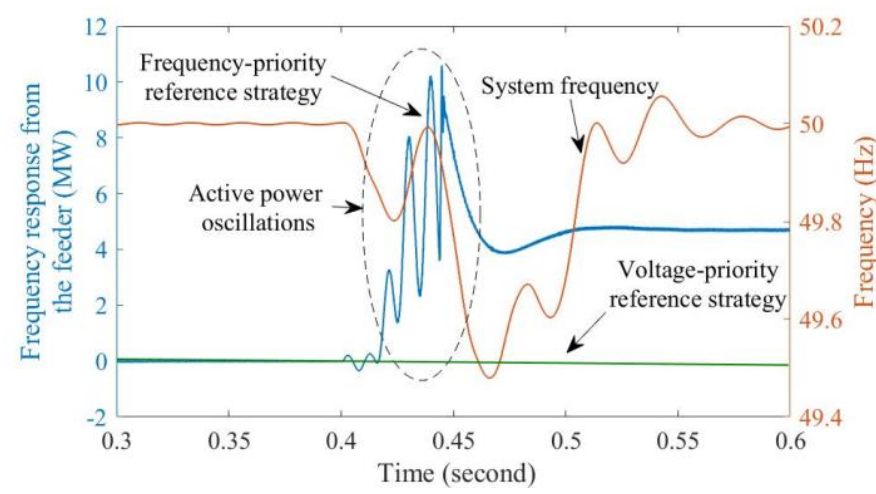

Fig. 13. System frequency dynamics as well as the battery frequency response in both voltage-priority and frequency-priority modes.

In Case-2, the battery gives full priority to reactive power support and delivers up to 5 MVar into the system (Fig. 14). This, in turn, prevented the frequency response provision from battery due to the limited converter capacity.

It is then of interest to evaluate the performance of the proposed voltage-priority approach in a worse condition with lower system strength. To do so, we apply the same fault (i.e., phase-to-phase-to-ground lasting for three frequency cycles) at the main substation at $\mathrm{t}=4 \mathrm{~s}$ while assuming $S C R_{D T P O I}=1$. The short-circuit fault results in around $67 \%$ voltage drop at the battery terminal (Fig. 15(a)). From the simulation results in Fig. 15, the proposed voltage-priority approach allocates the full battery converter capacity to deliver up to $5 \mathrm{MV}$ ar reactive power to the system (Fig. 15(b)). This helps the battery PLL to properly regulate the $q$-axis component of its terminal voltage, thereby resulting in PLL stability and battery synchronism.

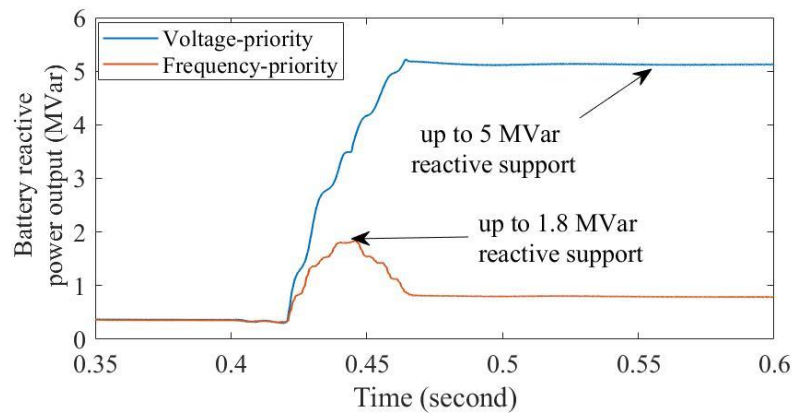

Fig. 14. Reactive power support from the distribution feeder in voltagepriority and frequency-priority cases. 

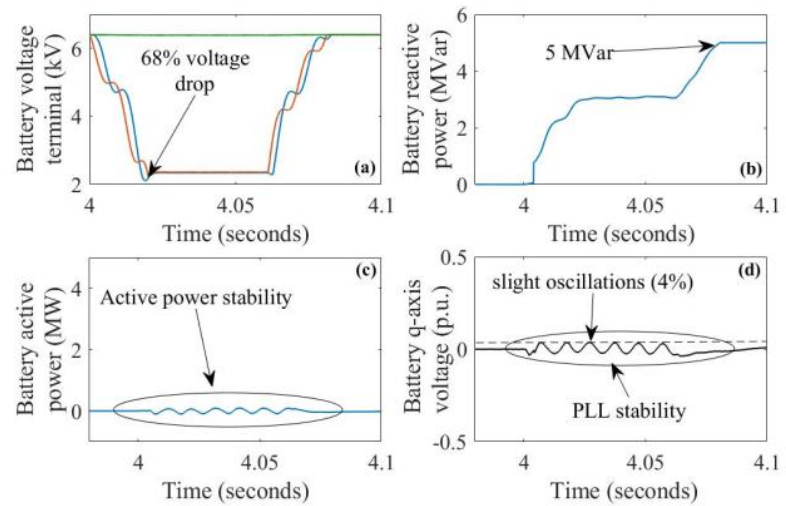

Fig. 15. Simulation results of the weak distribution grid under study assuming $S C R_{D T P O I}=1$ at the main substation: (a) severe voltage drop at the battery terminal, (b) stable reactive power support from the battery, (c) stable battery's active power output, (d) PLL stability.

It can be seen that the battery active power output is also stable (Fig. 15(c)), owing to converter-level control stability and battery synchronism obtained by the proposed voltagepriority approach. Further, the battery active power output is zero (Fig. 15(c)) since there is no more available converter capacity due to full reactive power support from the battery. This simulation study therefore demonstrates that the proposed voltage-priority approach is able to keep the battery active/reactive power outputs stable even in very low system strength levels (i.e., $S C R_{D T P O I}=1$ ).

Hence, two important points can be concluded from this section: 1) reactive power priority, as discussed in Section III$\mathrm{C}$, is an effective solution to enhance IBR stability in weak distribution grids, and 2) disturbance performance characteristics and related IBR active-reactive power interactions (like the ones we introduced in Section III-D) should be considered by system operators when defining frequency response requirements.

Finally, the following points can be considered as general values of the proposed voltage-priority approach itself: 1) Our proposed strategy suggests the need for further reviewing the analysis of converter-level control stability in order to consider IBR reactive power capability. This is since the converter control stability is typically discussed with focus on the stable operation of control loops and their impact on IBR active power control, but the converter's reactive power output and capability are ignored. 2) Our proposed strategy demonstrates the importance of $\left(P^{*}, Q^{*}\right)$ reference points in stable IBR synchronism and response. In fact, it shows that it is possible to achieve the IBR active/reactive power output stability and synchronism via proper reference point generation.

\section{CONCLUSIONS}

This paper introduces for the first time the modelling foundations to study IBR dynamics in weak distribution grids and define how they should react following small-/large-signal disturbances, while also proposing specific design requirements for their converter control. In the case of smallsignal disturbances, it has been discussed why weak distribution feeders are prone to simultaneous angle-voltage oscillations. Based on the presented mathematical discussions and also simulation results, there is a high chance of voltage violations following frequency response from IBRs, which is associated with low system strength at the DTPOI. These voltage violations may, in turn, prevent further frequency support from IBRs. Recommendations for fine-tuning of IBR $d-q$ current controllers are then provided as a potential solution to achieve a stable, desirable frequency response. Regarding the large-signal voltage disturbances, there is a high chance of poor IBR active power-voltage control due to a delay associated with the external system physical features (low system strength, resistive grid). On the contrary, reactive power control could be an effective solution to enhance IBR converter-level stability, i.e., PLL stability. Our simulation results show that our proposed voltage-priority reference generation strategy, which prioritizes reactive power over active power support during abnormal voltage conditions, is highly effective. However, this may prevent frequency response provision from IBRs due to their converter power transfer limit, which should be considered when defining frequency response guidelines and requirements.

\section{REFERENCES}

[1] S. Püschel-Løvengreen et al., "Separation event-constrained optimal power flow to enhance resilience in low-inertia power systems," Electric Power Systems Research, vol. 189, 2020.

[2] P. Mancarella and F. Billimoria, "The Fragile Grid: The Physics and Economics of Security Services in Low-Carbon Power Systems," IEEE Power and Energy Magazine, vol. 19, no. 2, pp. 79-88, 2021.

[3] M. Ghazavi Dozein et al., "System Strength and Weak Grids: Fundamentals, Challenges, and Mitigation Strategies," AUPEC 2018, pp. 1-7, 2018.

[4] M. Ghazavi Dozein et al., "Frequency Response From Solar PV: A Dynamic Equivalence Closed-Loop System Identification Approach," IEEE Systems Journal, 2021.

[5] M. Ghazavi Dozein, P. Mancarella, "Frequency response capabilities of utility-scale battery energy storage systems, with application to the August 2018 separation event in Australia", ICPES2019, pp. 1-6, 2019.

[6] M. Ghazavi Dozien, O. Gomis-Bellmunt, P. Mancarella, "Simultaneous Provision of Dynamic Active and Reactive Power Response from Utility-Scale Battery Energy Storage Systems in Weak Grids", IEEE Transactions on Power Systems, April 2021, in press.

[7] Fei Gao, Ren Kang, Jun Cao et al., "Primary and secondary control in DC microgrids: a review," Journal of Modern Power Systems and Clean Energy, vol. 7, no. 2, pp. 227-242, 2019.

[8] Yun Wang et al., "Cyber-physical power system modeling for timingdriven control of active distribution network," Journal of Modern Power Systems and Clean Energy, vol. 8, no. 3, pp. 549-556, 2020.

[9] R. Yan and T. K. Saha, "Investigation of Voltage Stability for Residential Customers Due to High Photovoltaic Penetrations," in IEEE Transactions on Power Systems, vol. 27, no. 2, pp. 651-662, 2012.

[10] F. J. Zimann et al., "Energy storage system control algorithm for voltage regulation with active and reactive power injection in low-voltage distribution network," Electric Power Systems Research, vol. 174, no. 105825, pp. 1-9, 2019.

[11] A. Jalali and M. Aldeen, "Risk-Based Stochastic Allocation of ESS to Ensure Voltage Stability Margin for Distribution Systems," IEEE Trans. on Power Systems, vol. 34, no. 2, pp. 1264-1277, 2019.

[12] M. Islam et al., "A Grid-Support Strategy with PV units to Boost Shortterm Voltage Stability under Asymmetrical Faults," IEEE Trans. on Power Systems, vol. 35, no. 2, pp. 1120-1131, 2020.

[13] I. I. Perpinias, N. P. Papanikolaou and E. C. Tatakis, "Optimum design of low-voltage distributed photovoltaic systems oriented to enhanced fault ride through capability," IET Generation, Transmission \& Distribution, vol. 9, no. 10, pp. 903-910, 272015. 
[14] Md. S. Ali et al., "A review of topological ordering based voltage rise mitigation methods for LV distribution networks with high levels of photovoltaic penetration," Renewable and Sustainable Energy Reviews, vol. 103, pp. 463-476, 2019.

[15] P. Aristidou, G. Valverde and T. Van Cutsem, "Contribution of Distribution Network Control to Voltage Stability: A Case Study," in IEEE Transactions on Smart Grid, vol. 8, no. 1, pp. 106-116, 2017.

[16] S. Mat Zali and J. V. Milanović, "Generic Model of Active Distribution Network for Large Power System Stability Studies," in IEEE Trans. on Power Systems, vol. 28, no. 3, pp. 3126-3133, Aug. 2013.

[17] J. Silva et al., "Estimating the Active and Reactive Power Flexibility Area at the TSO-DSO Interface," IEEE Transactions on Power Systems, vol. 33, no. 5, pp. 4741-4750, Sept. 2018.

[18] S. Riaz and P. Mancarella, "On Feasibility and Flexibility Operating Regions of Virtual Power Plants and TSO/DSO Interfaces," 2019 IEEE Milan PowerTech, pp. 1-6, 2019.

[19] S. Jafarpour, V. Purba, B. Johnson, S. Dhople and F. Bullo, "Singular Perturbation and Small-signal Stability for Inverter Networks," in IEEE Transactions on Control of Network Systems, 2021.

[20] P. Kundur, "Power system stability and control", McGraw-Hill Inc, New York, 1994.

[21] F. Díaz-González, A. Sumper, O. Gomis-Bellmunt, "Energy storage in power systems", John Wiley \& Sons publication, pp. 166-174, 2016.

[22] AEMC, "Inverstigation into system strength frameworks in the NEM, " pp. 1-128, 2020.

[23] A. Jalali, B. Badrzadeh, J. Lu, N. Modi, M. Gordon, "System strength challenges and solutions developed for a remote area of Australian power system with high penetration of inver-based resources, " CIGRE Science and Engineering Journal, no. 20, pp. 5-37, 2021.

[24] A. Engler, "Applicability of droops in low voltage grids," International Journal of Distributed Energy Resources, Germany, pp. 1-6, 2005.

[25] GHD advisory, "Managing system strength during the transition to renewables," GHD report for ARENA, 2020.

[26] NERC, "BPS-connected inverter-based resource performance," pp.1-98, 2018.

[27] X. He, H. Geng, R. Li and B. C. Pal, "Transient Stability Analysis and Enhancement of Renewable Energy Conversion System During LVRT," IEEE Trans. on Sustainable Energy, vol. 11, no. 3, pp. 1612-1623, 2020.

[28] AEMO, "Electricity rule change proposal, generator technical requirement", AEMO Information \& Support Hub, Australia, 2017.

[29] M. Gibbard and D. Vowles, "IEEE PES task force on benchmark systems for stability controls simplified 14-generator model of the south east Australian power system," 2014.

[30] AEMO, "2019 forecasting and planning scenarios, inputs, and assumptions", 2019.

[31] M. Ghazavi Dozein, A. Jalali and P. Mancarella, "Fast Frequency Response from Utility-Scale Hydrogen Electrolyzers," IEEE Transactions on Sustainable Energy, 2021. 